history of preterm delivery. Multiple logistic regression and generalised additive models were used to explore the effect of covariates including area deprivation, smoking status, BMI, parity and ethnicity. Results The proportion of PTB was significantly different in the three groups: $1.35 \%$ (95\% CI 1.24 to $1.47, \mathrm{n}=38994)$ in the low risk group, compared to $6.55 \%$ (CI 6.09 to $7.03, n=10760$ ) in the medical disorder group and $9.2 \%$ (CI 7.39 to $11.61, \mathrm{n}=732$ ) in the previous preterm group. $64 \%$ of the women delivering at LWH were in the most deprived quintile relative to the English population. The unadjusted odds of preterm delivery in the most deprived quintile compared to the least was 1.60 (CI 1.28 to 2.00) in the uncomplicated group. In a multiple regression model, ever having smoked (OR 1.68 CI 1.35 to 2.08), underweight (OR $1.65 \mathrm{CI} 1.005$ to 2.56) and highest quintile of area deprivation (OR 1.59 CI 1.19 to 2.11) were associated with increased the risk of PTB. Being overweight decreased the risk of PTB (OR 0.76 CI 0.59 to 0.97). In the medical disorders group, age (OR $1.02 \mathrm{CI} 1.011$ to 1.04), highest quintile of area deprivation (OR $1.46 \mathrm{CI} 1.14$ to 1.88 ), underweight (OR $1.68 \mathrm{CI}$ 1.09 to 2.51), ever having smoked (OR $1.19 \mathrm{CI} 1.00$ to 1.44), nulliparity (OR $1.37 \mathrm{CI} 1.13$ to 1.66 ) and black ethnic group (OR $1.61 \mathrm{CI}$ 1.00 to 2.48) were associated with PTB.

Conclusions Preterm delivery contributes to inequalities in infant mortality. In a cohort of women with no identifiable risk factors for PTB at booking, deprivation of area of residence is associated with higher risk of PTB, even with adjustment for smoking and underweight, which are also important independent risk factors. Deprivation of area of residence needs to be considered when comparing obstetric outcomes in units around the UK.

\section{P17 BIRTH SIZE DIFFERENCES BETWEEN WHITE AND PAKISTANI ORIGIN INFANTS BY GENERATION: RESULTS FROM THE BORN IN BRADFORD COHORT STUDY}

doi:10.1136/jech.2010.120477.17

${ }^{1} \mathrm{~J}$ West, ${ }^{2} \mathrm{D}$ A Lawlor, 'L Fairley, ${ }^{1} \mathrm{~J}$ Wright, ${ }^{3}$ for the Born in Bradford Research Group. ${ }^{1}$ Bradford Institute for Health Research, Bradford Hospitals NHS Trust, Bradford, UK; ${ }^{2}$ MRC Centre for Causal Analyses in Translational Epidemiology, University of Bristol, Bristol, UK; ${ }^{3}$ Born in Bradford Project, Bradford Institute for Health Research, Bradford Royal Infirmary, Bradford, UK

Background Previous studies have shown marked differences in birth weight between babies born in the UK of South Asian origin and those of UK origin. Whether such differences persist across generations in contemporary populations, the mechanisms underlying them and the extent to which other dimensions of birth size vary between these two groups is unclear.

Objective To describe differences in term birth weight, head, arm and abdominal circumference and skinfolds between Pakistani origin and white British origin infants and to investigate whether the magnitude of any differences reduces depending on whether the parents and grandparents of Pakistani infants are born in the UK or Pakistan.

Design Birth cohort study (born in Bradford (BiB)).

Setting Bradford, UK.

Participants 1838 white British and 2222 Pakistani mothers recruited to $\mathrm{BiB}$ who completed a questionnaire at 26 weeks gestation and their babies born between September 2007 and November 2009.

Main Outcome Measures Birth weight, head, arm and abdominal circumference and skinfolds.

Results Pakistani babies were lighter (mean difference $227.6 \mathrm{~g}, 95 \%$ CI 198.3 to 256.8), had smaller head, arm and abdominal circumferences (mean differences $0.43 \mathrm{~cm}, 95 \%$ CI 0.30 to $0.56 ; 0.22 \mathrm{~cm}$, $95 \%$ CI 0.10 to $0.34 ; 1.25 \mathrm{~cm}, 95 \%$ CI 1.02 to 1.39 , respectively) and smaller subscapular and triceps skinfold thickness (mean differences
$0.22 \mathrm{~mm}, 95 \%$ CI 0.12 to 0.32 and $0.21 \mathrm{~mm}, 95 \%$ CI 0.13 to 0.29 ) than white British infants. Differences remained significant following adjustment for deprivation. Mean birth weight was highest in Pakistani infants when both parents were born in Pakistan (3206 g) and was lowest when both parents were UK born (3165 g).

Conclusions These results reaffirm that significant differences in birth size exist between white British and Pakistani origin infants in the UK. Despite the assumption that differences will reduce over successive generations, mean birth weight has not increased in infants of UK born Pakistani origin parents compared with infants of Pakistani born parents. This suggests that differences may be genetically determined or are affected by epigenetic or persisting behaviour characteristics. Further analysis will include adjustment for additional socioeconomic variables, other maternal and family characteristics and birthplace of maternal and paternal grandparents.

\section{P18 CHILD MALTREATMENT CO-OCCURRENCE AND ASSOCIATIONS WITH HOUSEHOLD DYSFUNCTION: EVIDENCE FROM THE 1958 BRITISH BIRTH COHORT}

doi:10.1136/jech.2010.120477.18

${ }^{1} \mathrm{R}$ Denholm, ${ }^{1} \mathrm{C}$ Power, ${ }^{2} \mathrm{C}$ Thomas, ${ }^{1} \mathrm{~L}$ Li. ${ }^{1} \mathrm{M}$ RC Centre of Epidemiology for Child Health/Centre for Paediatric Epidemiology and Biostatistics, UCL Institute of Child Health, London, UK; ${ }^{2}$ Division of Community Health Sciences, St George's University of London, London, UK

Background Child maltreatment has been associated with adverse health outcomes, including risk of mental health problems and cardiovascular disease. Little is known about how different forms of maltreatment co-occur and whether different patterns are associated with household dysfunction. Delineation of co-occurrence is important to establish in order that long-term health outcomes can be better identified and understood.

Objective To investigate (1) to what extent specific maltreatment subtypes co-occur in a British birth cohort and (2) how these patterns were associated with household dysfunction.

Design Longitudinal survey; the 1958 British birth cohort.

Setting England, Scotland and Wales.

Participants Individuals born during 1 week in March 1958. At age $45 \mathrm{y}, 78 \%$ of the remaining cohort (11971) completed questions on childhood experiences.

Outcomes Child maltreatment before age 16, including psychological, physical and sexual abuse and witnessing intimate partner violence, collected at age $45 \mathrm{y}$. Eleven indicators of parental neglectful behaviour, collected at 7,11, 16 and $45 \mathrm{y}$, were aggregated to derive a cumulative neglect score. Information on household dysfunction (eg, parental mental health, alcohol/drug misuse, poverty) was collected during childhood and at $45 \mathrm{y}$. OR presented were adjusted for sex and social class at birth.

Results Psychological abuse $(10.0 \%)$ was the most commonly reported maltreatment, followed by physical abuse (6.1\%), witnessing abuse (6.0\%) and sexual abuse (1.6\%); $24 \%$ had a neglect score $\geq 3$. $14 \%$ of participants experienced any one subtype of abuse. Of these, almost two thirds (64\%) experienced further abuse subtypes and/or had a neglect score $\geq 3$. Witnessing or experiencing abuse increased odds of reporting another maltreatment, for example, psychological and physical abuse OR 37.9 (95\% CI 30.8 to 46.5). The odds of reporting any abuse increased with neglect score; for example, for sexual abuse OR ranged from 1.5 (0.9 to 2.6) to 4.5 (2.5 to 8.1). Common household dysfunction variables (eg, conflict and physical punishment) were strong predictors for all abuse subtypes. Other dysfunction measures most strongly associated with maltreatment differed, for example, odds of psychological abuse were increased in association with mother/father with 